TRANSACTIONS OF THE

AMERICAN MATHEMATICAL SOCIETY

Volume 363, Number 8, August 2011, Pages 4063-4079

S 0002-9947(2011)05083-X

Article electronically published on March 21, 2011

\title{
ABOUT BOUNDARY VALUES IN $A(\Omega)$
}

\author{
PIOTR KOT
}

\begin{abstract}
Assume that $\Omega \subset \mathbb{C}^{n}$ is a balanced bounded domain with a holomorphic support function (e.g. strictly pseudoconvex domain with $C^{2}$ boundary). We denote $\|f\|_{z}^{2}:=\int_{0}^{1}\left|f\left(e^{2 \pi i t} z\right)\right|^{2} d t$. Let $\varepsilon>0$ and $\sigma$ be a circular invariant Borel probability measure on $\partial \Omega$. If $g \in A(\Omega)$ and $h$ is a continuous function on $\partial \Omega$ with $|g|<h$ on $\partial \Omega$, then we construct nonconstant functions $f_{1}, f_{2} \in A(\Omega)$ with $\left\|g+f_{1}\right\|_{z} \leq\|h\|_{z},\left|\left(g+f_{2}\right)(z)\right| \leq \max _{|\lambda|=1} h(\lambda z)$ for $z \in \partial \Omega$ and

$$
\sigma\left(\left\{z \in \partial \Omega:\left\|g+f_{1}\right\|_{z} \neq\|h\|_{z} \vee \max _{|\lambda|=1}\left|\left(g+f_{2}\right)(\lambda z)\right| \neq \max _{|\lambda|=1} h(\lambda z)\right\}\right)<\varepsilon .
$$
\end{abstract}

Additionally if $\Omega$ is a circular, bounded, strictly convex domain with $C^{2}$ boundary, then we give the construction of $f_{3} \in \mathbb{O}(\Omega)$, the holomorphic function with: $\left\|h-\left|g+f_{3}^{*}\right|\right\|_{z}=0$ for all $z \in \partial \Omega$, where $f^{*}$ denotes the radial limit of $f$. We also construct $f_{4} \in A(\Omega)$ with $\left\|g+f_{4}\right\|_{z}=\|h\|_{z}$ for $z \in \partial \Omega$.

In all cases we can make $f_{i}$ arbitrarily small on a given compact subset $F \subset \Omega$ and make it vanish to a given order at the point 0 .

\section{INTRODUCTION}

In this paper we study a domain $\Omega \subset \mathbb{C}^{n}$ with a holomorphic support function on $\partial \Omega$ (e.g. $\Omega$ is a strictly pseudoconvex domain with $C^{2}$ boundary; see [2]). We shall say that $\Phi: \bar{\Omega} \times \partial \Omega \rightarrow \mathbb{C}$ is a holomorphic support function on $\partial \Omega$ if

(1) $\Phi(\cdot, z) \in A(\Omega)$.

(2) There exist constants $c_{1}, c_{2}>0$ such that for $z, w \in \partial \Omega$ we have

$$
\exp \left(-c_{2}\|z-w\|^{2}\right) \leq|\Phi(z, w)| \leq \exp \left(-c_{1}\|z-w\|^{2}\right) .
$$

(3) If $T$ is a compact subset of $\Omega$, then $\sup _{(z, w) \in T \times \partial \Omega}|\Phi(z, w)|<1$.

Let $\sigma$ be a Borel probability measure on $\partial \Omega$. We say that $\sigma$ is a circular invariant measure if $\sigma\left(e^{2 \pi i t} S\right)=\sigma(S)$ for $S$, any Borel set, in $\partial \Omega$ and $t \in \mathbb{R}$. In particular

$$
\int_{\partial \Omega} f\left(e^{2 \pi i t} z\right) d \sigma(z)=\int_{\partial \Omega} f(z) d \sigma(z)
$$

for $t \in \mathbb{R}$ and any $f$ - $\sigma$-measurable function on $\partial \Omega$.

We study $\|f\|_{z}:=\sqrt{\int_{0}^{1}\left|f\left(e^{2 \pi i t} z\right)\right|^{2} d t}$, the middle value of the holomorphic function $f$ on a circle given by the point $z \in \partial \Omega$. If $\sigma$ is a circular invariant Borel

Received by the editors December 16, 2007 and, in revised form, April 13, 2009.

2010 Mathematics Subject Classification. Primary 32A05, 32A40.

Key words and phrases. Maximum modulus set, inner function. 
probability measure and $f$ is a $\sigma$-measurable function on $\partial \Omega$, then we have $(1.1)$

$$
\|f\|_{\sigma}:=\sqrt{\int_{\partial \Omega}|f(z)|^{2} d \sigma(z)}=\sqrt{\int_{0}^{1} \int_{\partial \Omega}\left|f\left(e^{2 \pi i t} z\right)\right|^{2} d \sigma(z) d t}=\sqrt{\int_{\partial \Omega}\|f\|_{z}^{2} d \sigma(z)} .
$$

We say that a $K$ compact set is a maximum modulus set for $A(\Omega)$ if there exists $f \in A(\Omega)$ such that $|f|<1$ on $\bar{\Omega} \backslash K$ and $|f|=1$ on $K$. There is also a stronger concept of a peak set, when $f=1$ on $K$.

In fact it is well known that for $n>1$ a holomorphic nonconstant function $f \in A(\Omega)$ with $|f|=1$ on $\partial \Omega$ does not exist. Therefore maximum modulus sets and peak sets are extensively considered by many authors.

Topologically, peak sets and maximum modulus sets are small in strictly pseudoconvex domains, as was proved by Stout and Duchamp. The real topological dimension of a maximum modulus set is no more than $n$ [7] and for a peak set is no more than $n-1$ [8].

Let us observe that from the measure-theoretic point of view, peak sets and maximum modulus sets no longer have to be small. Stensönes Henriksen has proved 6 that every strictly pseudoconvex domain with $C^{\infty}$ boundary in $C^{n}$ has a peak set with a Hausdorff dimension $2 n-1$. However, Eric Löw has proved [5] the following fact about maximum modulus sets:

Theorem ([5, Theorem 4.5]). Let $\Omega \Subset \mathbb{C}^{n}$ be a strictly pseudoconvex domain with $C^{2}$ boundary, $\varepsilon>0$ and $\varphi$ a bounded lower semicontinuous function on $\partial \Omega$. Then there exists a nonconstant $f \in A(\Omega)$ such that $\operatorname{Re} f \leq \varphi$ on $\partial \Omega$ and $H^{2 n-1}(\{z \in \partial \Omega: \operatorname{Re} f(z) \neq \varphi(z)\})<\varepsilon$. If $\varphi>0$ there exists a nonconstant $g \in$ $A(\Omega)$ such that $|g| \leq \varphi$ on $\partial \Omega$ and $H^{2 n-1}(\{z \in \partial \Omega:|g(z)| \neq \varphi(z)\})<\varepsilon$.

Let us observe that if $g$ is from the above theorem, then the point $z \in \partial \Omega$ with $\|g\|_{z}=\|\varphi\|_{z}$ may not exist. For this reason, we investigate middle values of holomorphic functions.

\section{Boundary VAlues in $A(\Omega)$}

We start with the following fact:

Theorem ([2, Theorem 3.2]). Assume that there exists a holomorphic support function on $\partial \Omega$. Let $a \in(0,1)$. There exists a natural number $N_{1}=N_{1}(a, \partial \Omega)$ such that, if $\varepsilon \in(0,1), T$ is a compact subset of $\Omega, H$ is a continuous, strictly positive function on $\partial \Omega$, and $g$ is a complex continuous function on $\partial \Omega$, then there exist holomorphic functions $f_{1}, \ldots, f_{N_{1}} \in A(\Omega)$ such that $\left\|f_{j}\right\|_{T} \leq \varepsilon$ and

(1) $\left|\left(f_{j}+g\right)(z)\right|-|g(z)| \leq\left|f_{j}(z)\right|<H(z)$ for $j=1, \ldots, N_{1}$ and $z \in \partial \Omega$,

(2) $a H(z)<\max _{j=1, \ldots, N_{1}}\left|\left(f_{j}+g\right)(z)\right|-|g(z)|$ for $z \in \partial \Omega$.

The following lemma shows that in the above theorem it is possible to replace holomorphic functions with polynomials.

Lemma 2.1. Assume that $\Omega$ is a balanced bounded domain with a holomorphic support function on $\partial \Omega$. Let $a \in(0,1)$. There exists a natural number $N_{2}=$ $N_{2}(a, \partial \Omega)$ such that if $m \in \mathbb{N}, \varepsilon \in(0,1), T$ is a compact subset of $\Omega, H$ is a continuous, strictly positive function on $\partial \Omega$, and $g$ is a complex continuous function on $\partial \Omega$, then there exist polynomials $p_{1}, \ldots, p_{N_{2}}$ with $\left\|p_{j}\right\|_{T} \leq \varepsilon$ and

(1) $\left|p_{j}(z)\right|<H(z)$ for $j=1, \ldots, N_{2}$ and $z \in \partial \Omega$, 
(2) $a H(z)<\max _{j=1, \ldots, N_{2}}\left|\left(p_{j}+g\right)(z)\right|-|g(z)| \leq \max _{j=1, \ldots, N_{2}}\left|p_{j}(z)\right|$ for $z \in$ $\partial \Omega$

(3) $p_{j}$ is the sum of monomials of degree at least $m$.

Proof. Without loss of generality we can assume that $\max _{i=1, \ldots, n}\left|z_{i}\right| \geq 1$ for $z=$ $\left(z_{1}, \ldots, z_{n}\right) \in \partial \Omega$. Let $N_{1}$ be from [2, Theorem 3.2]. Now we can define $N_{2}=n N_{1}$. There exist continuous functions $\chi_{1}, \ldots, \chi_{n}, \widetilde{\chi}_{1}, \ldots, \widetilde{\chi}_{n}$ on $\partial \Omega$ such that

- $\left|\chi_{i}\right| \leq 1$ and $0 \leq \tilde{\chi}_{i}<1$ on $\partial \Omega$,

- $\chi_{i}(z)=\tilde{\chi}_{i}(z)=1$ for $\left|z_{i}\right| \geq 1$,

- $\chi_{i}(z)=z_{i}^{m}$ and $\tilde{\chi}_{i}=\left|z_{i}\right|^{m}$ for $\left|z_{i}\right| \leq \frac{1}{2}$,

- $\tilde{\chi}_{i}(z) \neq 0$ for $z_{i} \neq 0$.

Now due to [2, Theorem 3.2] we can construct functions $f_{j, i} \in A(\Omega)$ such that

- $\left|z_{i}^{m} f_{j, i}(z)\right|<\varepsilon$ for $z \in T$,

- $\left|z_{i}^{m} f_{j, i}(z)\right|<H(z) \widetilde{\chi}_{i}(z) \leq H(z)$ for $z \in \partial \Omega$,

- $a H(z) \widetilde{\chi}_{i}(z)<\max _{j=1, \ldots, N_{1}}\left|z_{i}^{m} f_{j, i}(z)+g(z) \chi_{i}(z)\right|-\left|g(z) \chi_{i}(z)\right|$ for $z \in \partial \Omega$.

If $z \in \partial \Omega$, then there exists a coordinate $z_{i}$ of $z=\left(z_{1}, \ldots, z_{n}\right)$ such that $\left|z_{i}\right| \geq 1$. Then $\chi_{i}(z)=\widetilde{\chi}_{i}(z)=1$ and

$$
a H(z)<\max _{j=1, \ldots, N_{1}}\left|z_{i}^{m} f_{j, i}(z)+g(z)\right|-|g(z)| .
$$

Now we can define $\widetilde{f}_{j, i}(z):=r^{m} z_{i}^{m} f_{j, i}(r z)$ for some $0<r<1$ in such a way that

- $\left\|\tilde{f}_{j, i}\right\|_{T}<\varepsilon$,

- $\left|\widetilde{f}_{j, i}(z)\right|<H(z)$ for $z \in \partial \Omega$,

- $a H(z)<\max _{j=1, \ldots, N_{1}, i=1, \ldots, n}\left|\tilde{f}_{j, i}(z)+g(z)\right|-|g(z)|$ for $z \in \partial \Omega$,

- $\tilde{f}_{j, i}$ is the sum of monomials of degree at least $m$.

Let us observe that $\widetilde{f}_{j, i}$ is a holomorphic function on some neighborhood of $\bar{\Omega}$. In particular $\widetilde{f}_{j, i}$ is the sum of homogeneous polynomials uniformly convergent on $\bar{\Omega}$. It implies that there exist polynomials $p_{1}, \ldots, p_{N_{2}}$ such that the properties (1)-(3) are fulfilled.

Lemma 2.2. Assume that $\Omega$ is a balanced bounded domain with a holomorphic support function on $\partial \Omega$. Let $\sigma$ be a circular invariant Borel probability measure on $\partial \Omega$. There exists $\gamma \in(0,1)$ such that if $g$ is a polynomial on $\mathbb{C}^{n}$ and $h$ is a continuous function on $\partial \Omega$ with $\|g\|_{z}<\|h\|_{z}$ for $z \in \partial \Omega$, then there exists a polynomial $f$ such that

(1) $|f(z)|<\sqrt{\|h\|_{z}^{2}-\|g\|_{z}^{2}}$ for $z \in \partial \Omega$,

(2) $\sqrt{\|g\|_{z}^{2}+\|f\|_{z}^{2}}=\|g+f\|_{z}<\|h\|_{z}$ for $z \in \partial \Omega$,

(3) $0<\|h\|_{\sigma}^{2}-\|g+f\|_{\sigma}^{2} \leq \gamma\left(\|h\|_{\sigma}^{2}-\|g\|_{\sigma}^{2}\right)$.

(4) Additionally we can make $f$ arbitrarily small on a given compact subset $F \subset \Omega$ and make it vanish to a given order at the point 0 .

Proof. Let $a, N_{2}$ be from Lemma 2.1 and $\gamma=1-\frac{a^{2}}{N_{2}}$. Due to Lemma 2.1 we can choose polynomials $p_{1}, \ldots, p_{N_{2}}$ such that

- $\left|p_{j}(z)\right|<\sqrt{\|h\|_{z}^{2}-\|g\|_{z}^{2}}$ for $z \in \partial \Omega$, 
- $a \sqrt{\|h\|_{z}^{2}-\|g\|_{z}^{2}}<\max _{j=1, \ldots, N_{2}}\left|p_{j}(z)\right|$ for $z \in \partial \Omega$,

- $p_{j}$ is the sum of monomials of degree at least $\operatorname{deg} g+1$.

Let $T_{j}:=\left\{z \in \partial \Omega: a \sqrt{\|h\|_{z}^{2}-\|g\|_{z}^{2}} \leq\left|p_{j}(z)\right|\right\}$. Since $\bigcup_{j=1}^{N_{2}} T_{j}=\partial \Omega$ there exists $j_{0}$ such that

$$
\int_{T_{j_{0}}}\left|p_{j_{0}}(z)\right|^{2} d \sigma(z) \geq \frac{a^{2}}{N_{2}} \int_{\partial \Omega}\|h\|_{z}^{2}-\|g\|_{z}^{2} d \sigma(z) .
$$

Let $f:=p_{j_{0}}$. The property (1) is obvious. Since $f$ is the sum of monomials of at least $\operatorname{deg} g+1$ degree, we can obtain the property (2)

$$
\|g+f\|_{z}^{2}=\|g\|_{z}^{2}+\|f\|_{z}^{2}<\|h\|_{z}^{2} .
$$

Moreover due to (2.1) we may estimate

$$
\begin{aligned}
\|f\|_{\sigma}^{2} & \geq \int_{\partial \Omega}\left|p_{j_{0}}(z)\right|^{2} d \sigma(z) \geq \int_{T_{j_{0}}}\left|p_{j_{0}}(z)\right|^{2} d \sigma(z) \\
& \geq \frac{a^{2}}{N_{2}} \int_{\partial \Omega}\|h\|_{z}^{2}-\|g\|_{z}^{2} d \sigma(z) \geq(1-\gamma)\left(\|h\|_{\sigma}^{2}-\|g\|_{\sigma}^{2}\right),
\end{aligned}
$$

which together with inequality (2.2) implies the property (3)

$$
\|h\|_{\sigma}^{2}-\|g+f\|_{\sigma}^{2}=\|h\|_{\sigma}^{2}-\|g\|_{\sigma}^{2}-\|f\|_{\sigma}^{2} \leq \gamma\left(\|h\|_{\sigma}^{2}-\|g\|_{\sigma}^{2}\right) .
$$

Since $f$ is a sum of polynomials from Lemma 2.1 we can easily make $f$ arbitrarily small on a given compact subset $F \subset \Omega$ and make it vanish to a given order at the point 0 .

Lemma 2.3. Assume that $\Omega$ is a balanced bounded domain with a holomorphic support function on $\partial \Omega$. Let $\varepsilon \in(0,1)$ and $\sigma$ be a circular invariant Borel probability measure on $\partial \Omega$. There exists $C>1$ such that if $g$ is a polynomial on $\mathbb{C}^{n}$ and $h$ is a continuous function on $\partial \Omega$ with $\|g\|_{z}<\|h\|_{z}$ for $z \in \partial \Omega$, then there exists a function $f \in A(\Omega)$ such that

- $\|g+f\|_{z} \leq\|h\|_{z}$ for $z \in \partial \Omega$,

- $\varepsilon|f(z)|^{2}<C\left(\|h\|_{\sigma}^{2}-\|g\|_{\sigma}^{2}\right)$ for $z \in \partial \Omega$,

- $\sigma\left(\left\{z \in \partial \Omega:\|g+f\|_{z}<\|h\|_{z}\right\}\right)<\varepsilon$.

- Additionally we can make $f$ arbitrarily small on a given compact subset $F \subset \Omega$ and make it vanish to a given order at the point 0 .

Proof. Without loss of generality we may assume that $|h| \leq 1$ on $\partial \Omega$. Let $\gamma \in(0,1)$ be from Lemma 2.2 and $M$ be such that $\frac{2}{M^{2}} \sum_{k=1}^{\infty} \frac{1}{k^{2}}=\varepsilon$. We construct the sequence of polynomials $f_{k}$ with the following properties:

(1) $g_{1}:=g, g_{k+1}:=g_{k}+f_{k}$,

(2) $\left\|g_{k}\right\|_{z}<\left\|h_{k}\right\|_{z}$ for $z \in \partial \Omega$,

(3) $c_{k}:=\sqrt{\left\|h_{k}\right\|_{\sigma}^{2}-\left\|g_{k}\right\|_{\sigma}^{2}}, c_{k+1}^{2} \leq \gamma c_{k}^{2}$,

(4) $h_{1}:=h, h_{k+1}(z):=\min \left\{\left\|h_{k}\right\|_{z}, \sqrt{\left\|g_{k}\right\|_{z}^{2}+M^{2} k^{2} c_{k}^{2}}\right\}$ for $z \in \partial \Omega$,

(5) $\left|f_{k}\right|<M k c_{k}$ on $\partial \Omega$. 
If $h_{k}, g_{k}$ are given (e.g. for $k=1$ ), then $h_{k+1}(z):=\min \left\{\left\|h_{k}\right\|_{z}, \sqrt{\left\|g_{k}\right\|_{z}^{2}+M^{2} k^{2} c_{k}^{2}}\right\}$. In particular $\left\|g_{k}\right\|_{z}<\left\|h_{k+1}\right\|_{z}$ for $z \in \partial \Omega$. Due to Lemma 2.2 there exists a polynomial $p$ such that

- $|p(z)|<\sqrt{\left\|h_{k+1}\right\|_{z}^{2}-\left\|g_{k}\right\|_{z}^{2}} \leq M k c_{k}$ for $z \in \partial \Omega$,

- $\sqrt{\left\|g_{k}\right\|_{z}^{2}+\|p\|_{z}^{2}}=\left\|g_{k}+p\right\|_{z}<\left\|h_{k+1}\right\|_{z}$ for $z \in \partial \Omega$,

- $\left\|h_{k+1}\right\|_{\sigma}^{2}-\left\|g_{k}+p\right\|_{\sigma}^{2} \leq \gamma\left(\left\|h_{k+1}\right\|_{\sigma}^{2}-\left\|g_{k}\right\|_{\sigma}^{2}\right) \leq \gamma\left(\left\|h_{k}\right\|_{\sigma}^{2}-\left\|g_{k}\right\|_{\sigma}^{2}\right)$.

In particular it is enough to choose $f_{k}:=p$ and observe that properties (1)-(5) are fulfilled.

Now we prove that it is enough to define $f=\sum_{k=1}^{\infty} f_{k}$. The property (3) implies $c_{k} \leq \gamma^{\frac{k-1}{2}} c_{1}$. Since

$$
\sum_{k=j}^{\infty}\left|f_{k}(z)\right| \leq \sum_{k=j}^{\infty} M k c_{k} \leq M c_{1} \sum_{k=j}^{\infty} k \gamma^{\frac{k-1}{2}}
$$

on $\partial \Omega$ we may conclude that $f \in A(\Omega)$.

Moreover we can choose $C=\max \left\{4 \sum_{k=1}^{\infty} \frac{1}{k^{2}}\left(\sum_{k=1}^{\infty} k \gamma^{\frac{k-1}{2}}\right)^{2}, 1\right\}$ and then

$$
\begin{aligned}
|f(z)|^{2} & \leq M^{2} c_{1}^{2}\left(\sum_{k=j}^{\infty} k \gamma^{\frac{k-1}{2}}\right)^{2} \leq \frac{2}{\varepsilon}\left(\sum_{k=1}^{\infty} \frac{1}{k^{2}}\right)\left(\|h\|_{\sigma}^{2}-\|g\|_{\sigma}^{2}\right)^{2}\left(\sum_{k=j}^{\infty} k \gamma^{\frac{k-1}{2}}\right)^{2} \\
& <\frac{C}{\varepsilon}\left(\|h\|_{\sigma}^{2}-\|g\|_{\sigma}^{2}\right)
\end{aligned}
$$

for $z \in \partial \Omega$. Let $V=\bigcup_{k=1}^{\infty} V_{k}$, where

$$
V_{k}:=\left\{z \in \partial \Omega: \sqrt{\left\|g_{k}\right\|_{z}^{2}+M^{2} k^{2} c_{k}^{2}}<\left\|h_{k}\right\|_{z}\right\} .
$$

If $\sigma\left(V_{k}\right)>\frac{1}{M^{2} k^{2}}$, then

$$
\begin{aligned}
c_{k}^{2} & =\left\|h_{k}\right\|_{\sigma}^{2}-\left\|g_{k}\right\|_{\sigma}^{2}=\int_{\partial \Omega}\left(\left\|h_{k}\right\|_{z}^{2}-\left\|g_{k}\right\|_{z}^{2}\right) d \sigma(z) \\
& \geq \int_{V_{k}} M^{2} k^{2} c_{k}^{2} d \sigma(z) \geq \sigma\left(V_{k}\right) M^{2} k^{2} c_{k}^{2}>c_{k}^{2},
\end{aligned}
$$

which is impossible, so we may conclude that $\sigma\left(V_{k}\right) \leq \frac{1}{M^{2} k^{2}}$ and

$$
\sigma(V) \leq \sum_{k=1}^{\infty} \sigma\left(V_{k}\right) \leq \sum_{k=1}^{\infty} \frac{1}{M^{2} k^{2}}=\frac{\varepsilon}{2}<\varepsilon .
$$

Let $\widetilde{h}=\lim _{k \rightarrow \infty} h_{k}$. We may observe that $h_{2}=\ldots=h_{k}=\ldots=\widetilde{h}$ on $\partial \Omega \backslash V$. Since $\lim _{k \rightarrow \infty} c_{k}=0$ and $\|g+f\|_{z} \leq\|\widetilde{h}\|_{z} \leq\|h\|_{z}$ for $z \in \partial \Omega$, then $\|g+f\|_{\sigma}=$ $\|\widetilde{h}\|_{\sigma}$ and

$$
\sigma\left(\left\{z \in \partial \Omega:\|g+f\|_{z}<\|h\|_{z}\right\}\right) \leq \sigma(V)<\varepsilon .
$$

Since $f$ is a sum of polynomials from Lemma 2.1 we can easily make $f$ arbitrarily small on a given compact subset $F \subset \Omega$ and such that it vanishes to a given order at the point 0 . 
Now we can prove our first main result.

Theorem 2.4. Assume that $\Omega$ is a balanced bounded domain with a holomorphic support function on $\partial \Omega$. Let $\varepsilon \in(0,1)$ and $\sigma$ be a circular invariant Borel probability measure on $\partial \Omega$. There exists $M>1$ such that for a given $g \in A(\Omega)$ and $h a$ continuous function on $\partial \Omega$ with $\|g\|_{z}<\|h\|_{z}$ for $z \in \partial \Omega$ there exists a function $f \in A(\Omega)$ such that

- $\|g+f\|_{z} \leq\|h\|_{z}$ for $z \in \partial \Omega$,

- $\sigma\left(\left\{z \in \partial \Omega:\|g+f\|_{z}<\|h\|_{z}\right\}\right)<\varepsilon$,

- $|f| \leq \frac{M}{\sqrt{\varepsilon}} \sqrt{\|h\|_{\sigma}^{2}-\|g\|_{\sigma}^{2}}$.

- Additionally we can make $f$ arbitrarily small on a given compact subset $F \subset \Omega$ and have it vanish to a given order at the point 0 .

Proof. Let $C>1$ be from Lemma 2.3. We construct a sequence of holomorphic functions $f_{m} \in A(\Omega)$ with the following properties:

(1) $h_{1}:=h, g_{0}=g, g_{m+1}:=g+\sum_{k=1}^{m+1} f_{k}$,

$$
h_{m+1}(z):=\min \left\{\left\|h_{m}\right\|_{z}, \sqrt{\left\|g_{m}\right\|_{z}^{2}+8^{-m}\left(\|h\|_{z}^{2}-\|g\|_{z}^{2}\right)}\right\} ;
$$

(2) $\left\|g_{m}\right\|_{z}<\left\|h_{m}\right\|_{z}$ for $z \in \partial \Omega$;

(3) $V_{m}:=\left\{z \in \partial \Omega:\left\|h_{m+1}\right\|_{z}<\left\|h_{m}\right\|_{z}\right\}$ and $\sigma\left(V_{m}\right)<\varepsilon 2^{-m-1}$;

(4) $\left|f_{m}\right| \leq 4 \sqrt{\frac{C}{\varepsilon}\left(\|h\|_{\sigma}^{2}-\|g\|_{\sigma}^{2}\right)} 2^{-m}$ on $\partial \Omega$.

Assume that $g_{0}, \ldots, g_{m-1}$ are given (e.g. for $m=1$ ). Since $\left\|g_{m-1}\right\|_{z}<\left\|h_{m}\right\|_{z}$ for $z \in \partial \Omega$, there exists $\delta \in(0,0.5)$ such that

$$
\begin{aligned}
\left\|h_{m}\right\|_{z}^{2} & <(1-2 \delta)^{2}\left\|h_{m}\right\|_{z}^{2}+8^{-m}\left(\|h\|_{z}^{2}-\|g\|_{z}^{2}\right), \\
\left\|h_{m}\right\|_{z} & >\left\|g_{m-1}\right\|_{z}+2 \delta\left\|h_{m}\right\|_{z}
\end{aligned}
$$

for $z \in \partial \Omega$. Since $\Omega$ is a balanced bounded domain, there exists a polynomial $q$ such that $\left|g_{m-1}-q\right|<\delta\left|h_{m}\right|$ on $\partial \Omega$. We can observe that $\|q\|_{z} \leq\left\|g_{m-1}\right\|_{z}+$ $\left\|q-g_{m-1}\right\|_{z}<\left\|g_{m-1}\right\|_{z}+\delta\left\|h_{m}\right\|_{z}<(1-\delta)\left\|h_{m}\right\|_{z}$ for $z \in \partial \Omega$. Now due to Lemma 2.3 there exists $f_{m} \in A(\Omega)$ such that

- $\left\|q+f_{m}\right\|_{z} \leq(1-\delta)\left\|h_{m}\right\|_{z}$ for $z \in \partial \Omega$,

- $\varepsilon 2^{-m-1}\left|f_{m}(z)\right|^{2}<C\left((1-\delta)^{2}\left\|h_{m}\right\|_{\sigma}^{2}-\|q\|_{\sigma}^{2}\right)$ for $z \in \partial \Omega$,

- $\sigma\left(\left\{z \in \partial \Omega:\left\|q+f_{m}\right\|_{z}<(1-\delta)\left\|h_{m}\right\|_{z}\right\}\right)<\varepsilon 2^{-m-1}$.

First we can observe the property $(2)$

$\left\|g_{m}\right\|_{z} \leq\left\|g_{m-1}+f_{m}\right\|_{z} \leq\left\|g_{m-1}-q\right\|_{z}+\left\|q+f_{m}\right\|_{z}<(\delta+1-\delta)\left\|h_{m}\right\|_{z} \leq\left\|h_{m}\right\|_{z}$.

The function $h_{m+1}$ is now defined by the property (1). Let $z \in \partial \Omega$ be such that $\left\|h_{m+1}\right\|_{z}<\left\|h_{m}\right\|_{z}$. In particular due to the definition of $h_{m+1}$ we have

$$
\left\|g_{m-1}+f_{m}\right\|_{z}^{2}<\left\|h_{m}\right\|_{z}^{2}-8^{-m}\left(\|h\|_{z}^{2}-\|g\|_{z}^{2}\right)<(1-2 \delta)^{2}\left\|h_{m}\right\|_{z}^{2}
$$

and

$$
\left\|q+f_{m}\right\|_{z} \leq\left\|q-g_{m-1}\right\|_{z}+\left\|g_{m-1}+f_{m}\right\|_{z}<(1-\delta)\left\|h_{m}\right\|_{z},
$$

so we may conclude the property (3). 
Let us observe that $\left\|h_{m}\right\|_{\sigma}^{2}-\left\|g_{m-1}\right\|_{\sigma}^{2} \leq 8^{-m+1}\left(\|h\|_{\sigma}^{2}-\|g\|_{\sigma}^{2}\right)$ for $m \geq 1$. Since $-\delta\left\|h_{m}\right\|_{\sigma}<\|q\|_{\sigma}-\left\|g_{m-1}\right\|_{\sigma}<\delta\left\|h_{m}\right\|_{\sigma}$ we may estimate

$$
\begin{aligned}
\frac{\varepsilon}{C 2^{m+1}}\left|f_{m}\right|^{2} & \leq(1-\delta)^{2}\left\|h_{m}\right\|_{\sigma}^{2}-\|q\|_{\sigma}^{2} \leq\left\|h_{m}\right\|_{\sigma}^{2}-\left\|g_{m-1}\right\|_{\sigma}^{2} \\
& \leq 8^{-m+1}\left(\|h\|_{\sigma}^{2}-\|g\|_{\sigma}^{2}\right)
\end{aligned}
$$

which implies the property (4): $\left|f_{m}\right| \leq 4 \sqrt{\frac{C}{\varepsilon}\left(\|h\|_{\sigma}^{2}-\|g\|_{\sigma}^{2}\right)} 2^{-m}$.

Now we define $M:=4 \sqrt{C} \sum_{m=1}^{\infty} 2^{-m}, f:=\sum_{k=1}^{\infty} f_{k}$ and $V=\bigcup_{k=1} V_{k}$. First we can observe that

$$
|f| \leq \sum_{m=1}^{\infty}\left|f_{m}\right| \leq \sum_{m=1}^{\infty} 4 \sqrt{\frac{C}{\varepsilon}\left(\|h\|_{\sigma}^{2}-\|g\|_{\sigma}^{2}\right)} 2^{-m} \leq \frac{M}{\sqrt{\varepsilon}} \sqrt{\|h\|_{\sigma}^{2}-\|g\|_{\sigma}^{2}},
$$

so we may conclude that $f \in A(\Omega)$. We have $h=h_{m}$ on $\partial \Omega \backslash V$ and $\sigma(V)<\varepsilon$. In particular $\|g+f\|_{z}=\|h\|_{z}$ for $z \in \partial \Omega \backslash V$ and $\|g+f\|_{z} \leq\|h\|_{z}$ for $z \in \partial \Omega$. Since $f$ is a sum of polynomials from Lemma 2.1 we can easily make $f$ arbitrarily small on a given compact subset $F \subset \Omega$ and such that it vanishes to a given order at the point 0 , which finishes the proof.

Our next application of Lemma 2.1 will be connected with a construction of maximal modulus sets with a large Hausdorff measure.

Theorem 2.5. Assume that $\Omega$ is a balanced bounded domain with a holomorphic support function on $\partial \Omega$. Let $\varepsilon \in(0,1)$ and $\sigma$ be a Borel probability measure on $\partial \Omega$. Let $g \in A(\Omega)$ and $h$ be a continuous, positive function on $\partial \Omega$ with $|g(z)|<h(z)=$ $h(\lambda z)$ for $z \in \partial \Omega$ and $|\lambda|=1$. Then there exists a function $f \in A(\Omega)$ such that $|g+f| \leq h$ on $\partial \Omega$ and

$$
\sigma\left\{z \in \partial \Omega: \max _{|\lambda|=1}|(g+f)(\lambda z)| \neq h(z)\right\}<\varepsilon .
$$

Additionally we can make $f$ arbitrarily small on a given compact subset $F \subset \Omega$ and make it vanish to a given order at the point 0 .

Proof. Let $a=\frac{1}{2}$ and $N_{0} \in \mathbb{N}$. We can choose $N_{2}=N_{2}(a, \partial \Omega)$ from Lemma 2.1. Let us denote $\omega(f)(z):=\max _{|\lambda|=1}|f(\lambda z)|, \gamma:=1-\frac{1}{4 N_{2}}$ and $M=\sum_{k=1}^{\infty} \frac{2}{\varepsilon k^{2}}$. We construct polynomials $f_{m}$ with the following properties:

(1) $g_{1}:=g, g_{k+1}:=g_{k}+f_{k}$;

(2) $\omega\left(g_{k}\right)<h_{k}$ on $\partial \Omega$;

(3) $c_{k}:=\int_{\partial \Omega} h_{k}-\omega\left(g_{k}\right) d \sigma, 0<c_{k+1} \leq \gamma c_{k}$;

(4) $h_{1}:=h, h_{k+1}:=\min \left\{h_{k}, \omega\left(g_{k}\right)+M k^{2} c_{k}\right\}$ on $\partial \Omega$;

(5) $\left|f_{k}\right| \leq M k^{2} c_{k}$ on $\partial \Omega$.

If $g_{1}, \ldots, g_{k}$ are given (e.g. for $k=1$ ), then $\omega\left(g_{k}\right)<h_{k+1}$ on $\partial \Omega$. Let

$$
\delta:=\frac{1}{8 N_{2}} \int_{\partial \Omega} h_{k+1}-\omega\left(g_{k}\right) d \sigma
$$

There exists $r \in(0,1)$ such that $\left|g_{k}(z)-g_{k}(r z)\right|<\delta$ for $z \in \partial \Omega$. Due to Lemma 2.1 there exist polynomials $p_{1}, \ldots, p_{N_{2}}$ such that

$$
\text { - }\left|p_{j}\right|<h_{k+1}-\omega\left(g_{k}\right) \leq M k^{2} c_{k} \text { on } \partial \Omega \text {, }
$$


- $\frac{1}{2}\left(h_{k+1}-\omega\left(g_{k}\right)\right)(z)<\max _{j=1, \ldots, N_{2}}\left(\left|g_{k}+p_{j}\right|-\left|g_{k}\right|\right)(z)$ for $z \in \partial \Omega$,

- $\left|p_{j}\right|<\delta$ on $r \bar{\Omega}$.

Due to $\left|p_{j}\right|<\delta$ on $r \partial \Omega$ we have

$$
\omega\left(g_{k}\right)(z) \leq \omega\left(g_{k}\right)(r z)+\delta<\omega\left(g_{k}+p_{j}\right)(r z)+2 \delta \leq \omega\left(g_{k}+p_{j}\right)(z)+2 \delta
$$

for $z \in \partial \Omega$. Since

$$
\left|g_{k}(z)\right|+\frac{1}{2}\left(h_{k+1}-\omega\left(g_{k}\right)\right)(z) \leq \max _{j=1, \ldots, N_{2}}\left|\left(g_{k}+p_{j}\right)(z)\right| \leq \max _{j=1, \ldots, N_{2}} \omega\left(g_{k}+p_{j}\right)(z),
$$

then

$$
\omega\left(g_{k}\right)(z)+\frac{1}{2}\left(h_{k+1}-\omega\left(g_{k}\right)\right)(z) \leq \max _{j=1, \ldots, N_{2}} \omega\left(g_{k}+p_{j}\right)(z)
$$

for $z \in \partial \Omega$. Let us define

$$
T_{i}:=\left\{z \in \partial \Omega: \max _{j=1, \ldots, N_{2}} \omega\left(g_{k}+p_{j}\right)(z)=\omega\left(g_{k}+p_{i}\right)(z)\right\} .
$$

Since $\bigcup_{i=1, \ldots, N_{2}} T_{i}=\partial \Omega$, there exists $i \in\left\{1, \ldots, N_{2}\right\}$ such that

$$
\int_{T_{i}} \frac{1}{2}\left(h_{k+1}-\omega\left(g_{k}\right)\right) d \sigma \geq \frac{1}{N_{2}} \int_{\partial \Omega} \frac{1}{2}\left(h_{k+1}-\omega\left(g_{k}\right)\right) d \sigma .
$$

We can define $f_{k}:=p_{i}$ and estimate

$$
\begin{aligned}
& \int_{\partial \Omega}\left(\omega\left(g_{k+1}\right)-\omega\left(g_{k}\right)\right) d \sigma \stackrel{2.4}{\geq} \int_{T_{i}} \frac{1}{2}\left(h_{k+1}-\omega\left(g_{k}\right)\right) d \sigma \\
& +\int_{\partial \Omega \backslash T_{i}}\left(\omega\left(g_{k+1}\right)-\omega\left(g_{k}\right)\right) d \sigma \\
& \stackrel{2.5}{\geq} \int_{T_{i}} \frac{1}{2}\left(h_{k+1}-\omega\left(g_{k}\right)\right) d \sigma+\int_{\partial \Omega \backslash T_{i}}-2 \delta d \sigma \\
& \stackrel{12.6}{\geq} \frac{1}{N_{2}} \int_{\partial \Omega} \frac{1}{2}\left(h_{k+1}-\omega\left(g_{k}\right)\right) d \sigma-2 \delta \\
& \stackrel{2.3}{\geq} \frac{1}{4 N_{2}} \int_{\partial \Omega}\left(h_{k+1}-\omega\left(g_{k}\right)\right) d \sigma .
\end{aligned}
$$

Now using the above inequality we may estimate

$$
\begin{aligned}
c_{k+1} & \leq \int_{\partial \Omega}\left(h_{k+1}-\omega\left(g_{k}\right)-\omega\left(g_{k+1}\right)+\omega\left(g_{k}\right)\right) d \sigma \\
& \leq \int_{\partial \Omega}\left(h_{k+1}-\omega\left(g_{k}\right)\right) d \sigma-\frac{1}{4 N_{2}} \int_{\partial \Omega}\left(h_{k+1}-\omega\left(g_{k}\right)\right) d \sigma \\
& \leq \gamma \int_{\partial \Omega}\left(h_{k+1}-\omega\left(g_{k}\right)\right) d \sigma \leq \gamma \int_{\partial \Omega}\left(h_{k}-\omega\left(g_{k}\right)\right) d \sigma \leq \gamma c_{k}
\end{aligned}
$$

Moreover due to $\left|g_{k}\right|+\left|f_{k}\right| \leq\left|g_{k}\right|+\left|p_{i}\right| \leq \omega\left(g_{k}\right)+\left|p_{i}\right|<h_{k+1}$ on $\partial \Omega$ we have $\omega\left(g_{k+1}\right)<h_{k+1}$, which implies that $0<c_{k+1}$ and $f_{k}$ have the required properties.

Let us define $f=\sum_{k=1}^{\infty} f_{k}$, where the polynomials $f_{k}$ have the properties (1)-(5).

The property (3) implies $c_{k} \leq \gamma^{k-1} c_{1}$. Since

$$
\sum_{k=j}^{\infty}\left|f_{k}\right| \leq \sum_{k=j}^{\infty} M k^{2} c_{k} \leq M c_{1} \sum_{k=j}^{\infty} k^{2} \gamma^{k-1}
$$

on $\partial \Omega$, we may conclude that $f \in A(\Omega)$. 
Let $V=\bigcup_{k=1}^{\infty} V_{k}$, where

$$
V_{k}:=\left\{z \in \partial \Omega: \omega\left(g_{k}\right)(z)+M k^{2} c_{k}<h_{k}(z)\right\} .
$$

If $\sigma\left(V_{k}\right)>\frac{1}{M k^{2}}$, then

$$
c_{k}=\int_{\partial \Omega}\left(h_{k}-\omega\left(g_{k}\right)\right) d \sigma \geq \int_{V_{k}} M k^{2} c_{k} d \sigma(z) \geq \sigma\left(V_{k}\right) M k^{2} c_{k}>c_{k},
$$

which is impossible, so we may conclude that $\sigma\left(V_{k}\right) \leq \frac{1}{M k^{2}}$ and

$$
\sigma(V) \leq \sum_{k=1}^{\infty} \sigma\left(V_{k}\right) \leq \sum_{k=1}^{\infty} \frac{1}{M k^{2}} \leq \frac{\varepsilon}{2}<\varepsilon
$$

We may observe that $h=h_{1}=\ldots=h_{k}$ on $\partial \Omega \backslash V$ and $\omega(g+f) \leq h$ on $\partial \Omega$. Due to $\lim _{k \rightarrow \infty} c_{k}=0$ we can obtain $\omega(g+f)=h$ on $\partial \Omega \backslash V$, which implies that $\sigma(\{z \in \partial \Omega: \omega(g+f)(z)<h(z)\})<\varepsilon$.

Since $f$ is a sum of polynomials from Lemma 2.1, we can easily make $f$ arbitrarily small on a given compact subset $F \subset \Omega$ and make it vanish to a given order at the point 0 .

2.1. Boundary values of $A\left(\Omega, \mathbb{C}^{K}\right)$. In this section we study holomorphic functions with complex vector values. If $g=\left(g_{1}, \ldots, g_{N}\right) \in A\left(\Omega, \mathbb{C}^{N}\right)$, then we denote $\|g\|_{z}:=\sqrt{\sum_{i=1}^{N}\left\|g_{i}\right\|_{z}^{2}}$ and $|g|=\sum_{i=1}^{N}\left|g_{i}\right|$ for $z \in \partial \Omega, m \in \mathbb{N}$. We say that $f=\left(f_{1}, \ldots, f_{N}\right) \in \mathbb{O}\left(\Omega, \mathbb{C}^{N}\right)$ is a vector of $N$ polynomials if $f_{1}, \ldots, f_{N}$ are polynomials. In such a case, $\operatorname{deg} f=\max \operatorname{deg}_{j=1, \ldots, N} f_{j}$.

Lemma 2.6. Assume that $\Omega$ is a balanced bounded domain with a holomorphic support function on $\partial \Omega$. There exist $K \in \mathbb{N}$ and $C>0$ such that if $g$ is a vector of $K$ polynomials and $h$ is a continuous function on $\partial \Omega$ with $\|g\|_{z}<\|h\|_{z}$ for $z \in \partial \Omega$, then there exists $f \in A\left(\Omega, \mathbb{C}^{K}\right)$ such that

(1) $|f(z)|<C \sqrt{\|h\|_{z}^{2}-\|g\|_{z}^{2}}$ for $z \in \partial \Omega$,

(2) $\|h\|_{z}=\|g+f\|_{z}$ for $z \in \partial \Omega$.

(3) Additionally we can make $f$ arbitrarily small on a given compact subset $F \subset \Omega$ and such that it vanishes to a given order at the point 0 .

Proof. Let $a \in(0,1)$ and $N_{2}$ be from Lemma 2.1. We denote $K=N_{2}, \gamma=\sqrt{1-\frac{a}{K}}$ and $C=\frac{\sqrt{K}}{1-\gamma}$. Due to Lemma 2.1 we can choose $f_{m}=\left(f_{1, m}, \ldots, f_{K, m}\right)$, a vector of $K$ polynomials such that

- $K\left|f_{j, m}(z)\right|^{2}<\|h\|_{z}^{2}-\left\|g_{m}\right\|_{z}^{2}$ for $z \in \partial \Omega$,

- $\frac{a}{K}\left(\|h\|_{z}^{2}-\left\|g_{m}\right\|_{z}^{2}\right)^{2}<\max _{j=1, \ldots, K}\left|f_{j, m}(z)\right|^{2}$ for $z \in \partial \Omega$,

- $f_{j, m}$ is the sum of monomials of at least $\max _{i=1, \ldots, K} \operatorname{deg} g_{m}+1$ degree,

where $g_{1}:=g$ and $g_{m+1}:=g_{m}+f_{m}$.

Let $f=\sum_{j=1}^{\infty} f_{j}$ and $z \in \partial \Omega$. We may observe that $\frac{a}{K}\left(\|h\|_{z}^{2}-\left\|g_{m}\right\|_{z}^{2}\right)<$ $\left\|f_{m}\right\|_{z}^{2}<\|h\|_{z}^{2}-\left\|g_{m}\right\|_{z}^{2}$ and

$$
0<\|h\|_{z}^{2}-\left\|g_{m+1}\right\|_{z}^{2} \leq\|h\|_{z}^{2}-\left\|g_{m}\right\|_{z}^{2}-\left\|f_{m}\right\|_{z}^{2}<\gamma^{2}\left(\|h\|_{z}^{2}-\left\|g_{m}\right\|_{z}^{2}\right) .
$$

In particular $\lim _{m \rightarrow \infty}\left\|g_{m}\right\|_{z}=\|h\|_{z}$ and

$$
K\left|f_{j, m}(z)\right|^{2}<\|h\|_{z}^{2}-\left\|g_{m}\right\|_{z}^{2}<\gamma^{2(m-1)}\left(\|h\|_{z}^{2}-\|g\|_{z}^{2}\right) .
$$


Since

$$
\sum_{j=1}^{K} \sum_{m=k}^{\infty}\left|f_{j, m}(z)\right| \leq \sqrt{K\left(\|h\|_{z}^{2}-\|g\|_{z}^{2}\right)} \sum_{m=k}^{\infty} \gamma^{m-1} \leq C \sqrt{\|h\|_{z}^{2}-\|g\|_{z}^{2}},
$$

we conclude that $f \in A\left(\Omega, \mathbb{C}^{K}\right)$ and $f$ fulfills the properties (1)-(2).

Since $f$ is a sum of vector polynomials constructed on the basis of Lemma 2.1, we can easily make $f$ arbitrarily small on a given compact subset $F \subset \Omega$ and such that it vanishes to a given order at the point 0 .

Theorem 2.7. Assume that $\Omega$ is a balanced bounded domain with a holomorphic support function on $\partial \Omega$. There exists $K \in \mathbb{N}$ such that if $g \in A\left(\Omega, \mathbb{C}^{K}\right)$ and $h$ is a continuous function on $\partial \Omega$ with $\|g\|_{z}<\|h\|_{z}$ for $z \in \partial \Omega$, then there exists a function $f \in A\left(\Omega, \mathbb{C}^{K}\right)$ such that $\|g+f\|_{z}=\|h\|_{z}$ for $z \in \partial \Omega$. Additionally we can make $f$ arbitrarily small on a given compact subset $F \subset \Omega$ and have it vanish to a given order at the point 0.

Proof. Let $K \in \mathbb{N}$ and $C>0$ be from Lemma 2.6. We construct a sequence of functions $f_{m} \in A\left(\Omega, \mathbb{C}^{K}\right)$ with the following properties:

(1) $0<\|h\|_{z}-\left\|g+\sum_{k=1}^{m} f_{k}\right\|_{z} \leq 2^{-2 m+2}\|h\|_{z}$ for $z \in \partial \Omega$,

(2) $\left|f_{m}(z)\right| \leq 2^{-m+3} C\|h\|_{z}$ for $z \in \partial \Omega$.

Let us define $f_{1}=0$. Assume that $f_{1}, \ldots, f_{m} \in A\left(\Omega, \mathbb{C}^{K}\right)$ and that the properties (1)-(2) are given (e.g. for $m=1$ ). Let us define

$$
\delta:=\frac{1}{2} \min _{w \in \partial \Omega}\left\{\|h\|_{w}-\left\|g+\sum_{k=1}^{m} f_{k}\right\|_{w}, 2^{-2 m}\|h\|_{w}\right\} .
$$

There exists $g_{m}$, a vector of $K$ polyomials, such that

$$
\left\|g_{m}-g-\sum_{k=1}^{m} f_{k}\right\|_{z}<\delta
$$

for $z \in \partial \Omega$. We may estimate

$$
\begin{aligned}
\|h\|_{z}-\left\|g_{m}\right\|_{z} & \leq\|h\|_{z}-\left\|g+\sum_{k=1}^{m} f_{k}\right\|_{z}+\left\|g_{m}-g-\sum_{k=1}^{m} f_{k}\right\|_{z} \\
& \leq 2\left(\|h\|_{z}-\left\|g+\sum_{k=1}^{m} f_{k}\right\| \|_{z}\right) \leq 2^{-2 m+3}\|h\|_{z} .
\end{aligned}
$$

Since

$$
\begin{aligned}
\left\|g_{m}\right\|_{z}+\delta & \leq\left\|g_{m}-g-\sum_{k=1}^{m} f_{k}\right\|_{z}+\left\|g+\sum_{k=1}^{m} f_{k}\right\|_{z}+\frac{1}{2}\left(\|h\|_{z}-\left\|g+\sum_{k=1}^{m} f_{k}\right\|{ }_{z}\right) \\
& <\|h\|_{z}-\left\|g+\sum_{k=1}^{m} f_{k}\right\|_{z}+\left\|g+\sum_{k=1}^{m} f_{k}\right\|_{z} \leq\|h\|_{z},
\end{aligned}
$$


we may apply Lemma 2.6 to $g_{m}$ and conclude that there exists $f_{m+1} \in A\left(\Omega, \mathbb{C}^{K}\right)$ such that $\left\|g_{m}+f_{m+1}\right\|_{z}=\|h\|_{z}-\delta$ and $f_{m+1}$ has the property (2)

$$
\begin{aligned}
\left|f_{m+1}(z)\right| & \leq C \sqrt{\|h\|_{z}^{2}-\left\|g_{m}\right\|_{z}^{2}} \leq C \sqrt{2\|h\|_{z}\left(\|h\|_{z}-\left\|g_{m}\right\|_{z}\right)} \\
& \leq C \sqrt{2\|h\|_{z} 2^{-2 m+3}\|h\|_{z}} \leq 2^{-m+2} C\|h\|_{z} .
\end{aligned}
$$

We may also observe that

$$
\begin{aligned}
\left\|g+\sum_{k=1}^{m+1} f_{k}\right\|_{z} & \leq\left\|g_{m}+f_{m+1}\right\|_{z}+\left\|g_{m}-g-\sum_{k=1}^{m} f_{k}\right\|_{z} \\
& <\|h\|_{z}-\delta+\delta \leq\|h\|_{z}
\end{aligned}
$$

and

$$
\begin{aligned}
\left\|g+\sum_{k=1}^{m+1} f_{k}\right\|_{z} & \geq\left\|g_{m}+f_{m+1}\right\|_{z}-\left\|g_{m}-g-\sum_{k=1}^{m} f_{k}\right\|_{z} \\
& \geq\|h\|_{z}-\delta-\delta \geq\|h\|_{z}-2 \delta \\
& \geq\left(1-2^{-2 m}\right)\|h\|_{z}
\end{aligned}
$$

for $z \in \partial \Omega$, which implies that $f_{m+1}$ fulfills the property (1).

Now we may define $f=\sum_{m=1}^{\infty} f_{m}$, where $f_{m} \in A\left(\Omega, \mathbb{C}^{K}\right)$ is the sequence of functions with properties (1)-(2). We can observe that $\sum_{m=k}\left|f_{m}(z)\right| \leq \sum_{m=k} \frac{C\|h\|_{z}}{2^{m-3}}$ for $z \in \partial \Omega$, which implies that $f \in A\left(\Omega, \mathbb{C}^{K}\right)$. Moreover we have $\|g+f\|_{z}=$ $\lim _{m \rightarrow \infty}\left\|g+\sum_{k=1}^{m} f_{k}\right\|_{z}=\|h\|_{z}$ for $z \in \partial \Omega$.

Since $f$ is a sum of vector polynomials constructed on the basis of Lemma 2.6 we can easily make $f$ arbitrarily small on a given compact subset $F \subset \Omega$ where it vanishes to a given order at the point 0 .

\section{INNER FUNCTIONS AND SOME GENERALIZATIONS}

We start with the following fact.

Theorem 3.1. Assume that $\Omega$ is a circular, bounded, strictly convex domain with $C^{2}$ boundary. There exists $K \in \mathbb{N}$ such that for $0<\epsilon<1$ and for each pair of compact, circular and disjoint sets $D, T$ such that $T \subset \partial \Omega, D \subset \partial \Omega$, we can choose $N_{0}=N_{0}(D, T, \epsilon) \in \mathbb{N}$ such that for each natural number $N \geq N_{0}$ and given $m_{1}, \ldots, m_{K}$ with $N \leq m_{1}<m_{2}<\ldots<m_{K} \leq 2 N$ there exist homogeneous polynomials $p_{m_{i}}$ with degree $m_{i}$ such that

(1) $\left|p_{m_{i}}(z)\right| \leq 2$ for all $z \in \partial \Omega$,

(2) $\sum_{k=1}^{K}\left|p_{m_{k}}(z)\right|^{2} \geq 0.25$ for all $z \in T$,

(3) $\sum_{k=1}^{K}\left|p_{m_{k}}(z)\right|^{2} \leq 2^{-(K m)^{1-\epsilon}}$ for all $z \in D$.

Proof. In [3, Theorem 2.6] we proved this result with the additional assumption that $m_{j}=m K+j$. We used [3, Lemma 2.5], which can also be applied in the case $N \leq m_{1}<m_{2}<\ldots<m_{K} \leq 2 N$. So in order to prove the required result, it is enough to repeat the same arguments as in the proof of [3, Theorem 2.6].

Now we can construct an inner function whose restriction to any slice is also an inner function. In fact we prove a stronger result. 
Theorem 3.2. Assume that $\Omega$ is a circular, bounded, strictly convex domain with $C^{2}$ boundary. Let $g \in A(\Omega)$ and $h$ be a strictly positive and lower semicontinuous function on $\partial \Omega$ with $|g(z)|<h(z)$ and $\|h\|_{z}<\infty$ for $z \in \partial \Omega$. Then there exists $f \in \mathbb{O}(\Omega)$ such that $\sup _{|\lambda|<1}|f(\lambda z)| \leq \max _{|\lambda|=1}(h+|g|)(\lambda z)$ and $\left\|h-\left|g+f^{*}\right|\right\|_{z}=0$ for $z \in \partial \Omega$, where $f^{*}$ denotes the radial limit of $f$. Additionally we can make $f$ arbitrarily small on a given compact subset $F \subset \Omega$ where it vanishes to a given order at the point 0.

Proof. Let $a=\frac{1}{2}$. We can define $\gamma=\frac{1}{768 N_{1}^{2} K^{2}}$, where $N_{1}=N_{1}(a)$ is a natural number from [2, Theorem 3.2] and $K$ is from Theorem 3.1. There exists a sequence of positive continuous functions $h_{m}$ on $\partial \Omega$ such that

$$
|g|<h_{1}<\ldots<h_{j}<h_{j+1}<\ldots<\lim _{m \rightarrow \infty} h_{m}=h
$$

on $\partial \Omega$. We construct a sequence of polynomials $q_{m}$ with the following properties:

(1) $q_{m+1}$ is the sum of monomials of degree at least $\operatorname{deg} q_{m}+1$,

(2) $\left|q_{m}\right| \leq 2^{-m}$ on $\left(1-2^{-m}\right) \bar{\Omega}$,

(3) $\left|g_{m}\right|<h_{m}$ on $\partial \Omega$,

(4) $\left\|g_{m+1}\right\|_{z}^{2}>\gamma\left\|h_{m}-\left|g_{m}\right|\right\|_{z}^{2}+\left\|g_{m}\right\|_{z}^{2}$ for $z \in \partial \Omega$,

where $g_{1}:=g$, and $g_{m+1}:=g+\sum_{k=1}^{m} q_{k}$.

Assume that $g_{m}$ is given (e.g. for $m=1$ ). Due to [2, Theorem 3.2] there exist functions $f_{0}, \ldots, f_{N_{1}-1} \in A(\Omega)$ such that

- $4 N_{1} K\left|f_{j}\right|<h_{m}-\left|g_{m}\right|$ on $\partial \Omega$,

- $\frac{1}{2}\left(h_{m}-\left|g_{m}\right|\right)<4 N_{1} K \max _{j}\left|f_{j}\right|$ on $\partial \Omega$.

Since $\Omega$ is a bounded balanced domain, then without loss of generality we can assume that $f_{0}, \ldots, f_{N_{1}-1}$ are polynomials and that there exists a polynomial $r_{m}$ such that

$$
\begin{aligned}
2\left\|r_{m}-g_{m}\right\|_{z}\left\|h_{m+1}\right\|_{z} & <\gamma\left\|h_{m}-\left|g_{m}\right|\right\|_{z}^{2} \\
\left|g_{m}(z)-r_{m}(z)\right| & <\frac{h_{m}(z)-\left|g_{m}(z)\right|}{2}
\end{aligned}
$$

for $z \in \partial \Omega$. Let $d=\max _{j} \operatorname{deg} f_{j}+1$. Due to Theorem 3.1, there exist: 11 $\max \left\{\operatorname{deg} q_{m-1}, \operatorname{deg} r_{m}\right\}$, a natural number large enough and homogeneous polynomials $p_{N+j d}$ of degree $N+j d$ with the following properties:

- $\left|p_{m}\right| \leq 2$ on $\partial \Omega$,

- $\sum_{j=0}^{K-1}\left|p_{m_{i, j}}\right|^{2} \geq \frac{1}{4}$ on $\partial \Omega$, where $m_{i, j}:=N+d(K i+j), i=0, \ldots, N_{1}-1$.

Now we can define

$$
q_{m}:=\sum_{i=0}^{N_{1}-1} f_{i} \sum_{j=0}^{K-1} p_{m_{i, j}} .
$$

The properties (1)-(2) are obvious for $N$ large enough.

We can estimate

$$
\left|q_{m}\right| \leq \sum_{i=0}^{N_{1}-1}\left|f_{i}\right| \sum_{j=0}^{K-1}\left|p_{m_{i, j}}\right|<\sum_{i=0}^{N_{1}-1} \frac{1}{4 N_{1} K}\left(h_{m}-\left|g_{m}\right|\right) \sum_{j=0}^{K-1} 2 \leq \frac{h_{m}-\left|g_{m}\right|}{2},
$$

which implies the property (3): $\left|g_{m+1}\right| \leq\left|g_{m}\right|+\left|q_{m}\right|<h_{m}<h_{m+1}$.

$$
{ }^{1} q_{0}:=0
$$


Let us choose $z \in \partial \Omega$. Due to $\left\{f_{i} p_{m_{i, j}}\right\}$ being a sequence of orthogonal polynomials, we can estimate

$$
\begin{aligned}
\left\|q_{m}\right\|_{z}^{2} & =\sum_{i=0}^{N_{1}-1} \sum_{j=0}^{K-1} \int_{0}^{1}\left|\left(f_{i} p_{m_{i, j}}\right)\left(e^{2 \pi i t} z\right)\right|^{2} d t \\
& =\sum_{i=0}^{N_{1}-1} \sum_{j=0}^{K-1}\left|p_{m_{i, j}}(z)\right|^{2} \int_{0}^{1}\left|f_{i}\left(e^{2 \pi i t} z\right)\right|^{2} d t \geq \sum_{i=0}^{N_{1}-1} \frac{1}{4} \int_{0}^{1}\left|f_{i}\left(e^{2 \pi i t} z\right)\right|^{2} d t \\
& \geq \frac{1}{256 N_{1}^{2} K^{2}} \int_{0}^{1}\left|\left(h_{m}-\left|g_{m}\right|\right)\left(e^{2 \pi i t} z\right)\right|^{2} d t \geq 3 \gamma\left\|h_{m}-\left|g_{m}\right|\right\|_{z}^{2} .
\end{aligned}
$$

Since $\left|r_{m}\right| \leq\left|g_{m}-r_{m}\right|+\left|g_{m}\right| \stackrel{3.2}{<} h_{m}<h_{m+1}$ and

$$
\begin{aligned}
\left|r_{m}+q_{m}\right| & \leq\left|r_{m}\right|+\left|q_{m}\right| \stackrel{\sqrt[3.2]{<}}{<}\left|g_{m}\right|+\frac{h_{m}-\left|g_{m}\right|}{2}+\left|q_{m}\right| \\
& \stackrel{3.3}{<}\left|g_{m}\right|+\frac{h_{m}-\left|g_{m}\right|}{2}+\frac{h_{m}-\left|g_{m}\right|}{2}=h_{m}<h_{m+1},
\end{aligned}
$$

we have

$$
\begin{aligned}
\left\|g_{m+1}\right\|_{z}^{2}-\left\|r_{m}+q_{m}\right\|_{z}^{2} & \geq\left(\left\|g_{m}+r_{m}\right\|_{z}-\left\|r_{m}+q_{m}\right\|_{z}\right)\left(\left\|g_{m+1}\right\|_{z}+\left\|r_{m}+q_{m}\right\|_{z}\right) \\
& \geq-\left\|r_{m}-g_{m}\right\|_{z} 2\left\|h_{m+1}\right\|_{z} \stackrel{3.1}{\geq}-\gamma\left\|h_{m}-\left|g_{m}\right|\right\|_{z}^{2}
\end{aligned}
$$

and

$$
\begin{aligned}
\left\|r_{m}\right\|_{z}^{2}-\left\|g_{m}\right\|_{z}^{2} & \geq-\left\|r_{m}-g_{m}\right\|_{z}\left(\left\|r_{m}\right\|_{z}+\left\|g_{m}\right\|_{z}\right) \\
& \geq-\left\|r_{m}-g_{m}\right\|_{z} 2\left\|h_{m+1}\right\|_{z} \frac{3.1}{\geq}-\gamma\left\|h_{m}-\left|g_{m}\right|\right\|_{z}^{2} .
\end{aligned}
$$

The last inequalities imply the property (4):

$$
\begin{aligned}
\left\|g_{m+1}\right\|_{z}^{2} & \geq\left\|r_{m}+q_{m}\right\|_{z}^{2}+\left\|g_{m+1}\right\|_{z}^{2}-\left\|r_{m}+q_{m}\right\|_{z}^{2} \\
& \geq\left\|r_{m}\right\|_{z}^{2}+\left\|q_{m}\right\|_{z}^{2}-\gamma\left\|h_{m}-\left|g_{m}\right|\right\|_{z}^{2} \\
& >\left\|q_{m}\right\|_{z}^{2}+\left\|g_{m}\right\|_{z}^{2}-2 \gamma\left\|h_{m}-\left|g_{m}\right|\right\|_{z}^{2} \\
& >\left\|g_{m}\right\|_{z}^{2}+\gamma\left\|h_{m}-\left|g_{m}\right|\right\|_{z}^{2} .
\end{aligned}
$$

In particular we have just proved that there exists a polynomial $q_{m}$ with the properties (1)-(4). Let us define the following holomorphic function:

$$
f=\sum_{m=1}^{\infty} q_{m}
$$

Moreover let us denote $f_{t}(z):=f(t z)$ and $q_{m, t}(z):=q_{m}(t z)$. Let us choose $z \in \partial \Omega$. We may estimate

$$
\begin{aligned}
\sup _{|\lambda|<1}|f(\lambda z)| & =\lim _{m \rightarrow \infty} \sup _{|\lambda|<1}\left|\sum_{k=1}^{m} q_{k}(\lambda z)\right| \leq \lim _{m \rightarrow \infty} \sup _{|\lambda|=1}\left|\left(g_{m+1}-g\right)(\lambda z)\right| \\
& \leq \sup _{|\lambda|=1}(h+|g|)(\lambda z) .
\end{aligned}
$$


Since $\left\|g_{m}\right\|_{z}<\left\|g_{m+1}\right\|_{z}<\left\|h_{m+1}\right\|_{z}<\|h\|_{z}<\infty$ there exists $\lim _{m \rightarrow \infty}\left\|g_{m}\right\|_{z}$, which implies that

$$
\lim _{m \rightarrow \infty} \gamma\left\|h_{m}-\left|g_{m}\right|\right\|_{z}^{2} \leq \lim _{m \rightarrow \infty}\left\|g_{m+1}\right\|_{z}-\lim _{m \rightarrow \infty}\left\|g_{m}\right\|_{z}=0 .
$$

In particular $\lim _{m \rightarrow \infty}\left\|h-\left|g_{m}\right|\right\|_{z}=0$.

Since $\left\{q_{m}\right\}$ is a sequence of orthogonal polynomials we have

$$
\sum_{k=1}^{\infty}\left\|q_{k}\right\|_{z}^{2}=\lim _{m \rightarrow \infty}\left\|\sum_{k=1}^{m} q_{k}\right\|_{z}^{2} \leq \lim _{m \rightarrow \infty}\left\|g_{m+1}-g\right\|_{z}^{2} \leq\|h+|g|\|_{z}^{2}
$$

and

$$
\lim _{t \rightarrow 1^{-}}\left\|g_{m}-g-f_{t}\right\|_{z}^{2}=\lim _{t \rightarrow 1^{-}}\left(\sum_{k=1}^{m-1}\left\|q_{k}-q_{k, t}\right\|_{z}^{2}+\sum_{k=m}^{\infty}\left\|q_{k, t}\right\|_{z}^{2}\right)=\sum_{k=m}^{\infty}\left\|q_{k}\right\|_{z}^{2} .
$$

Now we can obtain

$$
\begin{aligned}
\lim _{t \rightarrow 1^{-}}\left\|h-\left|g+f_{t}\right|\right\|_{z} & \leq \lim _{m \rightarrow \infty} \lim _{t \rightarrow 1^{-}}\left(\left\|h-\left|g_{m}\right|\right\|_{z}+\left\|\left|g_{m}\right|-\left|g+f_{t}\right|\right\|_{z}\right) \\
& \leq \lim _{m \rightarrow \infty} \lim _{t \rightarrow 1^{-}}\left(\left\|h-\left|g_{m}\right|\right\|_{z}+\left\|g_{m}-g-f_{t} \mid\right\|_{z}\right) \\
& \leq \lim _{m \rightarrow \infty}\left(\left\|h-\left|g_{m}\right|\right\|_{z}+\sqrt{\sum_{k=m}^{\infty}\left\|q_{k}\right\|_{z}^{2}}\right)=0,
\end{aligned}
$$

which implies that there exists $f^{*}\left(e^{2 \pi i t} z\right):=\lim _{s \rightarrow 1^{-}} f\left(s e^{2 \pi i t} z\right)$ for almost all $t \in$ $[0,1]$ and $\left\|h-\mid g+f^{*}\right\|_{z}=0$.

Since $f$ is a sum of polynomials constructed on the basis of Theorem 3.1 we can easily make $f$ arbitrarily small on a given compact subset $F \subset \Omega$ and have it vanish to a given order at the point 0 .

If $\Omega$ is a strictly convex domain, then we can improve the main result from section two.

Theorem 3.3. Assume that $\Omega$ is a circular, bounded, strictly convex domain with $C^{2}$ boundary. Let $g \in A(\Omega)$ and $h$ be a continuous function on $\partial \Omega$ with $\|g\|_{z}<\|h\|_{z}$ for $z \in \partial \Omega$. Then there exists $f \in A(\Omega)$ such that $\|g+f\|_{z}=\|h\|_{z}$ for $z \in \partial \Omega$. Additionally we can make $f$ arbitrarily small on a given compact subset $F \subset \Omega$ and such that it vanishes to a given order at the point 0.

Proof. Let $a=\frac{1}{2}$. We can define $\gamma=\frac{1}{768 N_{1}^{2} K^{2}}$, where $N_{1}=N_{1}(a)$ is a natural number from [2, Theorem 3.2] and $K$ is from Theorem 3.1. We construct a sequence of polynomials $q_{m}$ with the following properties:

(1) $q_{m+1}$ is the sum of monomials of degree at least $\operatorname{deg} q_{m}+1$,

(2) $\left|q_{m}(z)\right| \leq \sqrt{\|h\|_{z}^{2}-\left\|g_{m}\right\|_{z}^{2}}$ for $z \in \partial \Omega$,

(3) $\left\|g_{m}\right\|_{z}<\|h\|_{z}$ for $z \in \partial \Omega$,

(4) $\left\|g_{m+1}\right\|_{z}^{2}>\gamma\left(\|h\|_{z}^{2}-\left\|g_{m}\right\|_{z}^{2}\right)+\left\|g_{m}\right\|_{z}^{2}$ for $z \in \partial \Omega$,

where $g_{1}:=g$ and $g_{m+1}:=g+\sum_{k=1}^{m} q_{k}$. 
Assume that $g_{m}$ is given (e.g. for $m=1$ ). Due to [2, Theorem 3.2] there exist functions $f_{0}, \ldots, f_{N_{1}-1} \in A(\Omega)$ such that

- $4 N_{1} K\left|f_{j}(z)\right|<\sqrt{\|h\|_{z}^{2}-\left\|g_{m}\right\|_{z}^{2}}$ for $z \in \partial \Omega$,

- $\frac{1}{2} \sqrt{\|h\|_{z}^{2}-\left\|g_{m}\right\|_{z}^{2}}<4 N_{1} K \max _{j}\left|f_{j}(z)\right|$ for $z \in \partial \Omega$.

Since $\Omega$ is a bounded balanced domain and $g_{m} \in A(\Omega)$, then without loss of generality we can assume that $f_{0}, \ldots, f_{N_{1}-1}$ are polynomials and that there exists a polynomial $r_{m}$ such that

$$
\begin{aligned}
2\left\|r_{m}-g_{m}\right\|_{z}\|h\|_{z} & <\gamma\left(\|h\|_{z}^{2}-\left\|g_{m}\right\|_{z}^{2}\right) \\
\left\|g_{m}\right\|_{z}+\left\|r_{m}\right\|_{z} & <2\|h\|_{z}, \\
\left\|r_{m}\right\|_{z}^{2} & <\left\|g_{m}\right\|_{z}^{2}+\frac{\|h\|_{z}^{2}-\left\|g_{m}\right\|_{z}^{2}}{4} \\
\|h\|_{z} & >\left\|g_{m}-r_{m}\right\|_{z}+\sqrt{\frac{\|h\|_{z}^{2}+\left\|g_{m}\right\|_{z}^{2}}{2}}
\end{aligned}
$$

for $z \in \partial \Omega$. Let $d=\max _{j} \operatorname{deg} f_{j}+1$. Due to Theorem 3.1 there exist:2 $N>$ $\max \left\{\operatorname{deg} q_{m-1}, \operatorname{deg} r_{m}\right\}$, a natural number large enough, and homogeneous polynomials $p_{N+j d}$ of degree $N+j d$ with the following properties:

- $\left|p_{m}\right| \leq 2$ on $\partial \Omega$,

- $\sum_{j=0}^{K-1}\left|p_{m_{i, j}}\right|^{2} \geq \frac{1}{4}$ on $\partial \Omega$, where $m_{i, j}:=N+d(K i+j), i=0, \ldots, N_{1}-1$.

Now we can define

$$
q_{m}:=\sum_{i=0}^{N_{1}-1} f_{i} \sum_{j=0}^{K-1} p_{m_{i, j}} .
$$

The property (1) is obvious.

We can estimate

$$
\begin{aligned}
\left|q_{m}(z)\right| & \leq \sum_{i=0}^{N_{1}-1}\left|f_{i}(z)\right| \sum_{j=0}^{K-1}\left|p_{m_{i, j}}(z)\right|<\sum_{i=0}^{N_{1}-1} \frac{\sqrt{\|h\|_{z}^{2}-\left\|g_{m}\right\|_{z}^{2}}}{4 N_{1} K} \sum_{j=0}^{K-1} 2 \\
& \leq \frac{\sqrt{\|h\|_{z}^{2}-\left\|g_{m}\right\|_{z}^{2}}}{2},
\end{aligned}
$$

which implies the property (2). Let us choose a $z \in \partial \Omega$. Since $r_{m}$ and $q_{m}$ are orthogonal polynomials we may observe

$$
\left\|r_{m}+q_{m}\right\|_{z}^{2}=\left\|r_{m}\right\|_{z}^{2}+\left\|q_{m}\right\|_{z}^{2} \stackrel{\sqrt{3.6], 33.8}}{<}\left\|g_{m}\right\|_{z}^{2}+\frac{\|h\|_{z}^{2}-\left\|g_{m}\right\|_{z}^{2}}{2} \leq \frac{\|h\|_{z}^{2}+\left\|g_{m}\right\|_{z}^{2}}{2},
$$

which implies the property (3)

$$
\begin{aligned}
\left\|g_{m+1}\right\|_{z} & =\left\|g_{m}+q_{m}\right\|_{z} \leq\left\|g_{m}-r_{m}\right\|_{z}+\left\|r_{m}+q_{m}\right\|_{z} \\
& \leq\left\|g_{m}-r_{m}\right\|_{z}+\sqrt{\frac{\|h\|_{z}^{2}+\left\|g_{m}\right\|_{z}^{2}}{2}} \frac{\sqrt{3.7}}{<}\|h\|_{z} .
\end{aligned}
$$

\footnotetext{
${ }^{2} q_{0}:=0$
} 
Due to $\left\{f_{i} p_{m_{i, j}}\right\}$ being a sequence of orthogonal polynomials, we may estimate

$$
\begin{aligned}
\left\|q_{m}\right\|_{z}^{2} & =\sum_{i=0}^{N_{1}-1} \sum_{j=0}^{K-1} \int_{0}^{1}\left|\left(f_{i} p_{m_{i, j}}\right)\left(e^{2 \pi i t} z\right)\right|^{2} d t \\
& =\sum_{i=0}^{N_{1}-1} \sum_{j=0}^{K-1}\left|p_{m}(z)\right|^{2} \int_{0}^{1}\left|f_{i}\left(e^{2 \pi i t} z\right)\right|^{2} d t \geq \sum_{i=0}^{N_{1}-1} \frac{1}{4} \int_{0}^{1}\left|f_{i}\left(e^{2 \pi i t} z\right)\right|^{2} d t \\
& \geq \frac{\|h\|_{z}^{2}-\left\|g_{m}\right\|_{z}^{2}}{256 N_{1}^{2} K^{2}} \geq 3 \gamma\left(\|h\|_{z}^{2}-\left\|g_{m}\right\|_{z}^{2}\right) .
\end{aligned}
$$

We may estimate

$$
\begin{aligned}
\left\|g_{m+1}\right\|_{z}^{2}-\left\|r_{m}+q_{m}\right\|_{z}^{2} & =\left(\left\|g_{m}+q_{m}\right\|_{z}-\left\|r_{m}+q_{m}\right\|_{z}\right)\left(\left\|g_{m+1}\right\|_{z}+\left\|r_{m}+q_{m}\right\|_{z}\right) \\
& \geq-\left\|r_{m}-g_{m}\right\|_{z} 2\|h\|_{z} \stackrel{(3.4)}{>}-\gamma\left(\|h\|_{z}^{2}-\left\|g_{m}\right\|_{z}^{2}\right)
\end{aligned}
$$

and

$$
\begin{gathered}
\left\|r_{m}\right\|_{z}^{2}-\left\|g_{m}\right\|_{z}^{2}=\left(\left\|r_{m}\right\|_{z}-\left\|g_{m}\right\|_{z}\right)\left(\left\|r_{m}\right\|_{z}+\left\|g_{m}\right\|_{z}\right) \\
\geq \quad-\left\|r_{m}-g_{m}\right\|_{z}\left(\left\|g_{m}\right\|_{z}+\left\|r_{m}\right\|_{z}\right) \stackrel{3.5}{\geq}-\left\|r_{m}-g_{m}\right\|_{z} 2\|h\|_{z} \\
\frac{3.4}{>}-\gamma\left(\|h\|_{z}^{2}-\left\|g_{m}\right\|_{z}^{2}\right) .
\end{gathered}
$$

The above three inequalities imply the property (4)

$$
\begin{aligned}
\left\|g_{m+1}\right\|_{z}^{2} & =\left\|r_{m}+q_{m}\right\|_{z}^{2}+\left\|g_{m+1}\right\|_{z}^{2}-\left\|r_{m}+q_{m}\right\|_{z}^{2} \\
& \geq\left\|r_{m}\right\|_{z}^{2}+\left\|q_{m}\right\|_{z}^{2}-\gamma\left(\|h\|_{z}^{2}-\left\|g_{m}\right\|_{z}^{2}\right) \\
& >\left\|g_{m}\right\|_{z}^{2}+\left\|r_{m}\right\|_{z}^{2}-\left\|g_{m}\right\|_{z}^{2}+2 \gamma\left(\|h\|_{z}^{2}-\left\|g_{m}\right\|_{z}^{2}\right) \\
& >\left\|g_{m}\right\|_{z}^{2}+\gamma\left(\|h\|_{z}^{2}-\left\|g_{m}\right\|_{z}^{2}\right) .
\end{aligned}
$$

In particular we have just proved that there exist polynomials $q_{m}$ with the properties (1)-(4). Let us define the following holomorphic function:

$$
f=\sum_{m=1}^{\infty} q_{m}
$$

We may observe that

$$
\|h\|_{z}^{2}-\left\|g_{m+1}\right\|_{z}^{2}<\|h\|_{z}^{2}-\gamma\left(\|h\|_{z}^{2}-\left\|g_{m}\right\|_{z}^{2}\right)-\left\|g_{m}\right\|_{z}^{2} \leq(1-\gamma)\left(\|h\|_{z}^{2}-\left\|g_{m}\right\|_{z}^{2}\right) \text {. }
$$

In particular

$$
\|h\|_{z}^{2}-\left\|g_{m}\right\|_{z}^{2}<(1-\gamma)^{m-1}\left(\|h\|_{z}^{2}-\left\|g_{1}\right\|_{z}^{2}\right)=(1-\gamma)^{m-1}\left(\|h\|_{z}^{2}-\|g\|_{z}^{2}\right) .
$$

Now we have

$$
\sum_{m=k}\left|q_{m}(w)\right| \leq \sum_{m=k} \sqrt{\|h\|_{w}^{2}-\left\|g_{m}\right\|_{w}^{2}} \leq \sum_{m=k}(1-\gamma)^{\frac{m-1}{2}} \sqrt{\|h\|_{w}^{2}-\|g\|_{w}^{2}}
$$


for $w \in \partial \Omega$, which implies that $f \in A(\Omega)$. Since $\left\|g_{m}\right\|_{z}<\left\|g_{m+1}\right\|_{z}<\|h\|_{z}$ there exists $\lim _{m \rightarrow \infty}\left\|g_{m}\right\|_{z}$ and

$$
0 \leq \lim _{m \rightarrow \infty} \gamma\left(\|h\|_{z}^{2}-\left\|g_{m}\right\|_{z}^{2}\right) \leq \lim _{m \rightarrow \infty}\left\|g_{m+1}\right\|_{z}^{2}-\lim _{m \rightarrow \infty}\left\|g_{m}\right\|_{z}^{2}=0 .
$$

Now we can obtain that $\|g+f\|_{z}=\lim _{m \rightarrow \infty}\left\|g_{m}\right\|_{z}=\|h\|_{z}$.

Since $f$ is a sum of polynomials constructed on the basis of Theorem 3.1, we can easily make $f$ arbitrarily small on a given compact subset $F \subset \Omega$ such that it vanishes to a given order at the point 0 .

Note. In Theorem 2.5 we assumed that $\Omega$ is a balanced bounded domain with a holomorphic support function on $\partial \Omega$. If we add more information on $\Omega$, e.g. that $\Omega$ is a circular, bounded, strictly convex domain with $C^{2}$ boundary, then this result can also be improved.

Theorem (4, Theorem 2.7]). Assume that $\Omega$ is a circular, bounded, strictly convex domain with $C^{2}$ boundary. Let $\varepsilon>0, T$ be a compact subset of $\Omega, g$ be a complex continuous function on $\partial \Omega$ and $h$ be a positive continuous function on $\partial \Omega$ such that $|g(z)|<h(z)=h(\lambda z)$ for $|\lambda|=1, z \in \partial \Omega$. Then there exists $f \in A(\Omega)$ such that $\|f\|_{T}<\varepsilon$ and $\max _{|\lambda|=1}|(g+f)(\lambda z)|=h(z)$ for $z \in \partial \Omega$.

\section{REFERENCES}

[1] A.B. Aleksandrov: The existence of inner functions in the unit ball. Math. Sb. 117, 147-163 (1982). MR658785 (83i:32002)

[2] P. Kot: A Holomorphic Function with Given Almost All Boundary Values on a Domain with Holomorphic Support Function, Journal of Convex Analysis 14, no. 4, 693-704 (2007). MR2350811(2008m:32009)

[3] P. Kot: Homogeneous polynomials on strictly convex domains, Proc. Amer. Math. Soc. 135, 3895-3903 (2007). MR2341939 (2008m:32010)

[4] P. Kot: Bounded holomorphic functions with given maximum modulus on all circles, Proc. Amer. Math. Soc. 137, 179-187 (2009). MR2439439 (2009f:32008)

[5] E. Löw: Inner Functions and Boundary Values in $H^{\infty}(\Omega)$ and $A(\Omega)$ in Smoothly Bounded Pseudoconvex Domains, Math. Z. 185, 191-210 (1984). MR731340 (85d:32032)

[6] B. Stensönes Henriksen: A peak set of Hausdorff dimension $2 n-1$ for the algebra $A(D)$ in the boundary of a domain $D$ with $C^{\infty}$-boundary in $\mathbb{C}^{n}$, Math. Ann. 259, 271-277 (1982). MR.656666 (83k:32028)

[7] E.L. Stout, Th. Duchamp: Maximum modulus sets, Ann. Inst. Fourier (Grenoble) 31.3, 37-69 (1981). MR638616 (83d:32019)

[8] E.L. Stout: The dimension of peak interpolation sets, Proc. Amer. Math. Soc. 86, no. 3, 413-416 (1982). MR671206 (84e:32015)

Instytut Matematyki, Politechnika Krakowska, ul. Warszawska 24, 31-155 Kraków, POLAND

E-mail address: pkot@pk.edu.pl 